\title{
Abordaje multidisciplinar de úlceras neuroisquémicas; a propósito de un caso
}

\author{
Multidisciplinary approach to neuroischaemic ulcers; a case report \\ Eduardo Simón Pérez ${ }^{1}$, Miguel Ángel Mellado Sanz², José Ignacio Rodríguez Mateos ${ }^{3}$, David Alonso Peña ${ }^{3}$, \\ Clarisa Simón Pérez ${ }^{4}$ y Celia Miñón Santamaría ${ }^{3}$
}

${ }^{1}$ Clínica Médico-Quirúrgica Recoletas Paracelso. Valladolid, España. ${ }^{2}$ Heridea Enfermería Avanza, Palencia, España. ${ }^{3}$ Servicio de Cirugía Plástica, Estética y Reparadora. Hospital Universitario Río Hortega. Valladolid, España. ${ }^{4}$ Servicio de Cirugía Ortopédica y Traumatología. Hospital Clínico Universitario. Valladolid, España

Palabras clave:

Pie diabético, abordaje multidisciplinar, úlceras neuroisquémicas, diabetes mellitus, terapia de presión negativa, factores de crecimiento, K-laser Cube 4, fillet toe, amputación de Syme.

Keywords:

Diabetic foot, multidisciplinary approach, neuroischaemic ulcers, diabetes mellitus, negative pressure wound therapy, growth factors, K-laser Cube 4, fillet toe, Syme amputation.

\section{Resumen}

Las úlceras neuroisquémicas son las lesiones más predominantes en el pie diabético en las que tanto la neuropatía como la isquemia contribuyen a esta manifestación clínica. Es una enfermedad de elevada prevalencia y morbilidad en nuestro medio, especialmente en el pie diabético. En este artículo se expone el caso de un paciente con una amputación del tercer dedo del pie izquierdo no resuelta tras un año de evolución. El paciente recibió una propuesta de amputación transmetatarsiana como solución para su problema. Él rechazó esa posibilidad y decidió buscar una segunda opinión. La colaboración de distintos profesionales especialistas en cirugía vascular, cirugía plástica, enfermería y podología, unido a técnicas novedosas como la terapia de presión negativa o curas con factores de crecimiento, permitieron la preparación del lecho para una cobertura exitosa, evitando una amputación transmetatarsiana. Las técnicas quirúrgicas que se realizaron fueron una amputación parcial de la falange distal del primer dedo (syme) como tratamiento de la osteomielitis que sufría en la falange distal y un fillet flap en el segundo dedo que cubrió el defecto de la lesión producida por la amputación, y posterior evolución tórpida del tercer dedo. Este tipo de técnicas pueden ser muy resolutivas si se aplican con una indicación correcta y pueden evitar tratamientos más agresivos que pueden condicionar la calidad de vida del paciente.

\begin{abstract}
Neuroischemic ulcers are the most prevalent lesions found in the diabetic foot since both neuropathy and ischemia contribute to this clinical manifestation. It's a high prevalence and morbidity disease. This article report the case of a patient with a $3^{\text {rd }}$ left toe amputation unsolved after one year treatment. The patient received a proposal for transmetatarsal amputation as a solution to their problem. He rejected that possibility and decided to look for a second opinion. Collaboration of different professionals specialists in vascular surgery, plastic surgery, nursing and podiatry, associated to innovative techniques such as negative pressure wound therapy or cures with growth factors allowed the wound for successful coverage avoiding transmetatarsal amputation. The surgical techniques performed were a partial amputation of the distal phalanx of the first toe (syme) as a treatment of osteomyelitis suffering in the distal phalanx and a fillet flap on the second finger that covered the defect of the injury caused by amputation, and subsequent tow-up evolution, of the third finger. Such techniques can be very resolute if applied with a correct indication and can avoid more aggressive treatments that can condition the quality of life of the patient.
\end{abstract}




\section{INTRODUCCIÓN}

El pie diabético es una de las complicaciones crónicas de la diabetes que suponen una mayor carga económica y social ${ }^{1,2}$. Implica entre el 28 y el $47 \%$ de las estancias hospitalarias de los pacientes diabéticos. Cada año, en el mundo, 4 millones de diabéticos desarrolla una úlcera en el pie, aproximadamente un $15 \%$ del total ${ }^{3-5}$.

Según los estudios, el 15 \% de las úlceras desarrollarán una osteomielitis que conlleve a una amputación; de estos, el $50 \%$ muere en, aproximadamente, 5 años ${ }^{1,4,6-8}$.

Los datos que se manejaban antiguamente eran que las úlceras más frecuentes eran las neuropáticas, entre un 45 y un $60 \%$ del total, las neuroisquémicas entre un 25 y un $45 \%$, y que las puramente isquémicas, que constituyen la manifestación final de la isquemia arterial periférica crónica ${ }^{9}$, entre un 10 y un $15 \%{ }^{10}$. Sin embargo, en la actualidad se sabe que, en nuestro medio, hasta el $50 \%$ de los pacientes con diabetes y úlcera en el pie presentan enfermedad arterial periférica (EAP) subyacente, por lo que la mayoría de las úlceras del pie son neuroisquémicas, ya que tienen un componente mixto de neuropatía e isquemia ${ }^{11}$, aunque pueda predominar la neuropatía y, por lo tanto, en los pacientes con úlceras neuroisquémicas, a pesar de tener isquemia severa del pie, los síntomas pueden estar ausentes debido a la presencia de esta neuropatía 2,12,13.

El manejo multidisciplinar supone una mejoría de los resultados objetivos así como de la percepción del paciente sobre su enfermedad ${ }^{14,15}$, y juega un papel importante para reducir y mantener una baja incidencia de amputaciones importantes en pacientes diabéticos, como demostró un estudio retrospectivo de Larsson y cols. en el que la incidencia de amputaciones mayores disminuyó en un 78 \% gracias al abordaje multidisciplinar de dichos pacientes ${ }^{16}$. A continuación se presenta un caso que ejemplifica este manejo, en el que intervinieron profesionales de campos como la cirugía vascular, la cirugía plástica, la enfermería y la podología con el objetivo común de evitar una amputación mayor en un paciente varón de 83 años con isquemia crítica de extremidad inferior izquierda (EII) secundaria a arteriopatía diabética, con lesiones tróficas en primer dedo y a nivel de 3 articulación metatarsofalángica (AMTF).

Para la redacción del artículo de este caso clínico seguimos las directrices de la guía CARE ${ }^{17}$.

\section{CASO CLÍNICO}

Paciente de 83 años seguido en el servicio de cirugía vascular por arteriopatía diabética e isquemia crítica de extremidad inferior izquierda de 17 años de evolución (EII), con angioplastia de las arterias femoral y poplítea y revascularización con stent en miembro inferior izquierdo que se llevó a cabo en noviembre de 2017. Se realizó tras presentar una lesión necrótica en el pulpejo del tercer dedo (Figura 1). A su vez, se amputó la falange distal del mismo dedo pero su evolución fue tórpida y conllevó posteriormente a una amputación
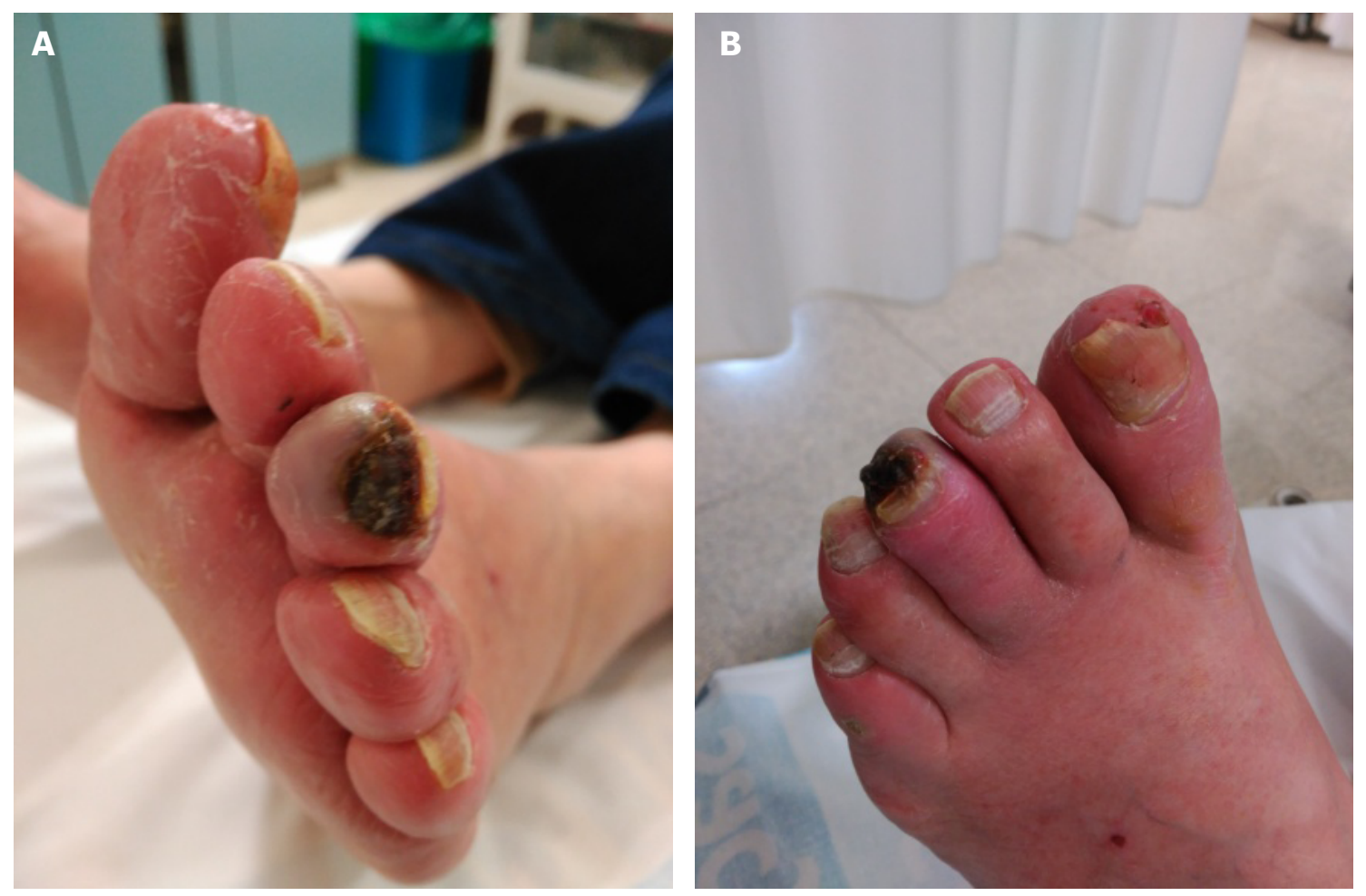

Figura 1. Pie diabético Wagner 3. A. Visión plantar. B. Visión dorsal. 
abierta del tercer dedo del pie izquierdo a nivel metatarsofalángico que se realizó el 1 diciembre de 2017, todo ello dentro del servicio de cirugía vascular del mismo hospital.

El paciente no refería alergias medicamentosas conocidas. Entre los antecedentes destacaba: exfumador desde hacía 12 años, dislipemia, diabetes mellitus insulinodependiente con buenos controles (HbA1c 6,5\%), hipotiroidismo, polimialgia reumática y pancreatitis aguda de origen biliar tratada con esfincterotomía laparoscópica, infarto de miocardio inferoposterior en 2010 con función ventricular conservada y revascularizado con stent.

En julio de 2018 ingresó de nuevo en el servicio de cirugía vascular por dehiscencia de muñón de amputación de falange distal de 3 dedo de Ell (realizada en diciembre de 2017), con abundante fibrina y exudado purulento, muy dolorosa a la palpación, por lo que se programó una intervención en la que se realizó Friedrich, que consiste en la eliminación del tejido esfacelado o necrótico de una úlcera por medios quirúrgicos ${ }^{18}$, y una resección de la cabeza del tercer metatarsiano, según informe. La evolución fue tórpida con curas y antibioterapia y se propuso amputación transmetatarsiana que el paciente rechazó y fue dado de alta con curas semanales en su centro de atención primaria (CAP) con colagenasa (Iruxol mono ${ }^{\circledR}$, Smith\&Newphew, España).

Tras varias curas durante dos meses en su CAP sin evolución y con gran dolor, decidió buscar una segunda opinión. Acudió a consulta privada de enfermería especializada en heridas el 2 de septiembre de 2018 y en la exploración presentó lesión en la zona distal del hallux, y el lecho de amputación, que se extiendía por el 2. ${ }^{\circ}$ dedo, estaba esfacelado y maloliente, sugestivo de infección por bacterias gram negativas polimicrobianas y con anaerobios, por lo que se informó del caso al podólogo del equipo multidisciplinar y se decidió pautar tratamiento antibiótico empírico (levofloxacino 750 mg + clindamicina 300 cada 8 h) tras la recogida de muestras para cultivo (Figura 2).
A nivel vascular, el pulso femoral y poplíteo estaba conservado con ausencia de pulso pedio y tibial posterior. Se realizó un índice tobillo-brazo con valor de 1,23 que indujo a pensar en una calcificación arterial, por lo que ese valor fue considerado como poco fiable. En este momento el paciente refería un dolor 9 según escala de EVA ${ }^{19}$.

Dada la ausencia de flujo arterial directo al pie, se decidió tratamiento conservador inicial y se programaron curas con limpieza exhaustiva con fomentos de una solución de súper oxidación (Mycrodacin ${ }^{\circledR}$, more Pharma, México) y posterior cura tópica con agua purificada, 0,1\% undecilenamidopropil betaína, 0,1\% polihexanida (Prontosan ${ }^{\circledR}$, B/Braun, España); se realizó curetaje del lecho y se observó la exposición de la primera falange y de la articulación interfalángica proximal (IFP) del $2 .^{\circ}$ dedo, por lo que se llevó a cabo la cura con mechas de fibras hidrodetersivas + alginato + espuma cada 24 h durante 10 días.

Tras la evolución favorable del lecho, se inició terapia de presión negativa ambulatoria (Pico ${ }^{\circledR}$, Smith\&Newphew, España) durante tres semanas (Figura 3), que generó la neoformación de tejido de granulación y la contracción del lecho por el uso de esta terapia negativa ${ }^{20}$. Se modificó la pauta de curas con la utilización de un factor de crecimiento epidérmico en gel ${ }^{21}$ (EPIFACTOR ${ }^{\circledR}$, Fagron, España) cada 48 h. Se observó la reducción del edema y del dolor (valoración de 5 en EAV) y no se apreciaron signos de infección durante los 7 días en los que se realizaron estas curas, por lo que se informó de esta evolución al podólogo que realizó una consulta conjunta con el cirujano plástico para la valoración del abordaje quirúrgico.

Se realiza la valoración del paciente el 10 de octubre de 2018, en la que se destaca: la ausencia de pulso pedio y tibial posterior (ya documentada anteriormente), ausencia de las sensibilidades algésica (se valoró mediante un pin-prick), barestésica (se valoró mediante un monofilamento de Semmes-Weinstein) y palestésica (se valoró mediante un diapa-
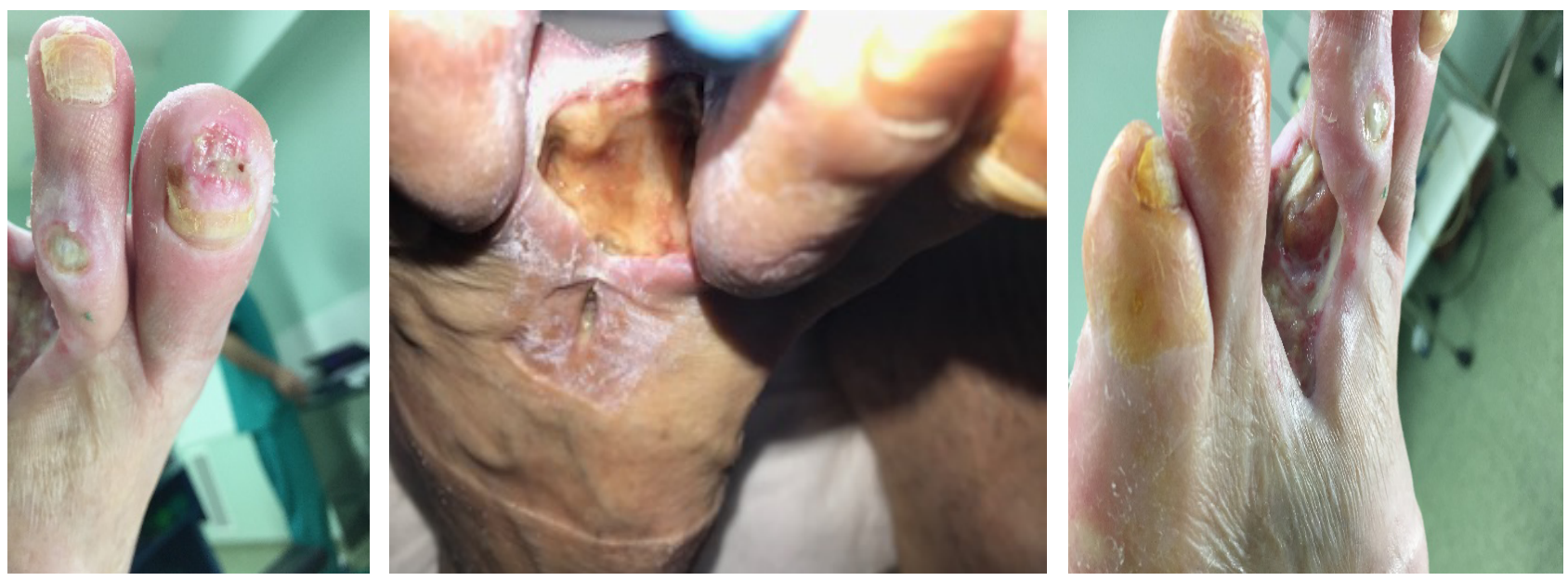

Figura 2. Lecho de amputación esfacelado y sugestivo de infección a la llegada a la consulta. 


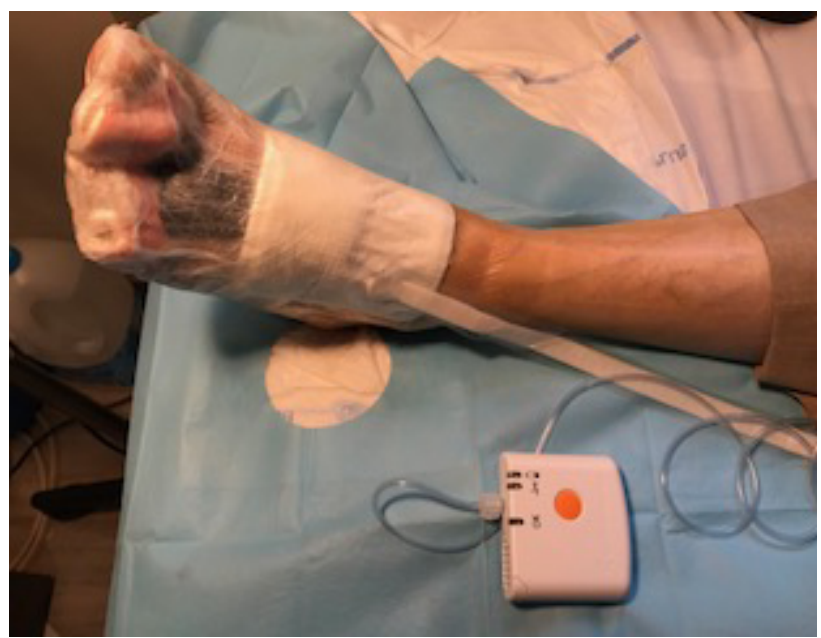

Figura 3. Terapia de presión negativa ambulatoria $\left(\mathrm{Pico}^{\circledR}\right) \mathrm{con}$ apósitos de acetato con tecnología DACC.

són), y una lesión esfacelada que ocupaba toda la zona de la amputación del tercer dedo y se extendía por el lateral del $2 .^{\circ}$ dedo; también presentaba una lesión en el pulpejo del primer dedo. Ambas lesiones tenían un probe to bone test positivo que realizamos con un mosquito curvo y nos hizo sospechar de osteomielitis ${ }^{22}$

Hicimos radiografía anteroposterior en carga y oblicua del pie izquierdo en la que apreciamos cambios sugestivos de osteomielitis a nivel de la falange distal del hallux y la articulación interfalángica proximal del $2 .^{\circ}$ dedo. En la 3. ${ }^{a}$ cabeza metatarsal los signos radiológicos no eran concluyentes de osteomielitis, ya que le habían realizado un Friedrich en esta zona y los daños óseos podrían haber sido provocados por esa intervención, aunque la clínica era compatible con osteomielitis.

Concluimos que el diagnóstico era úlceras neuroisquémicas a nivel de 3 , a articulación metatarsofalángica, extendiéndose hasta el borde lateral de $2{ }^{\circ}$ dedo y en la zona distal del hallux del pie izquierdo.

El paciente estaba en tratamiento con insulina glarfina 100 unidades/ml (Lantus ${ }^{\circledR}$, Sanofi, España), clopidrogel, besilato 75 mg (Clopidrogel ${ }^{\circledR}$, Tarbis, España) ácido acetilsalicílico (AAS) 100 mg (Adiro ${ }^{\circledR}$, Bayer, Alemania), ranitidina 150 (Ranitidina ${ }^{\circledR}$, Cinfa, España), enoxaparina sódica $60 \mathrm{mg}$ (Clexane ${ }^{\circledR}$, Sanofi Aventis, España), levotiroxina de sodio 100 $\mu \mathrm{g}$ (Eutirox ${ }^{\circledR}$, Merck, España), enalapril maleato 5 mg (Enalapril $^{\circledR}$, Sandoz, España) y atorvastatina 20 (Atorvastatina ${ }^{\circledR}$, Sandoz, España).

Al día siguiente, después de una puesta en común entre cirugía plástica, cirugía vascular y podología, se decidió proponer al paciente, que ya había rechazado una amputación transmetatarsiana, la opción de realizar un amputación atípica distal ${ }^{23}$, que consistía en realizar una amputación de syme en el primer dedo y una técnica de fillet toe del $2 .^{\circ}$ dedo que

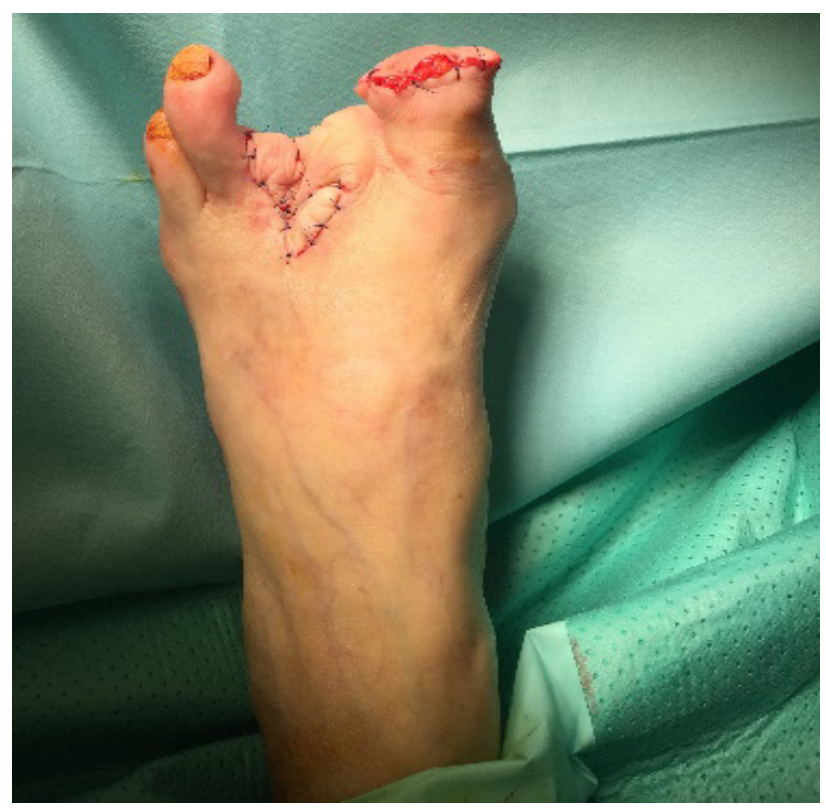

Figura 4. Resultado postoperatorio inmediato tras cobertura mediante colgajo fillet toe y amputación de falange distal del primer dedo.

consiguiera tapar el defecto de la lesión que afectaba a la 3. ${ }^{\text {a }}$ articulación metatarsofalángica, ya que desde cirugía vascular rechazaban la posibilidad de realizar otra revascularización. El paciente, entendiendo los riesgos de la intervención y las posibles complicaciones que podían, incluso, suponer una amputación mayor por su afectación vascular, aceptó el tratamiento. Por su doble antiagregación (AAS 100 mg/24 h y copidogrel $75 \mathrm{mg} / 24 \mathrm{~h}$ ), y además estando en tratamiento con enoxaparina, decidimos pasar a una antiagregación simple y suspender el copidogrel 5 días antes de la intervención y la enoxaparina se retiró 1 día antes de la intervención ${ }^{24}$, que se programó y se realizó el 17 de octubre de 2018.

La intervención quirúrgica se llevó a cabo bajo anestesia local con mepivacaína 2 \% (Mepivacaína ${ }^{\circledR}$, BBraun, España) y sin realización de isquemia. Se realizó una técnica de "fillet toe" del segundo dedo, que sufría artritis séptica en la articulación metatarsofalángica y en la articulación interfalángica proximal, para realizar la cobertura del defecto en el tercer espacio $^{25}$. Esta técnica de fillet toe consiste en la utilización lateral del colgajo de un dedo menor del pie, en el que se eliminan todas las falanges, en este caso afectadas por osteomielitis, y se conserva la piel y el tejido celular subcutáneo conservando su vascularización, y se usa como pedículo o colgajo libre microvascular para la reconstrucción de un defecto adyacente. Es una técnica reproducible que incurre en una complejidad técnica comparativamente mínima y proporciona una opción favorable en el tratamiento de las heridas del pie diabético donde se requiere cobertura de tejidos blandos $^{25}$. En el primer dedo se realizó amputación de syme ${ }^{26}$ para el tratamiento de osteomielitis de la falange distal (Figura 4), 

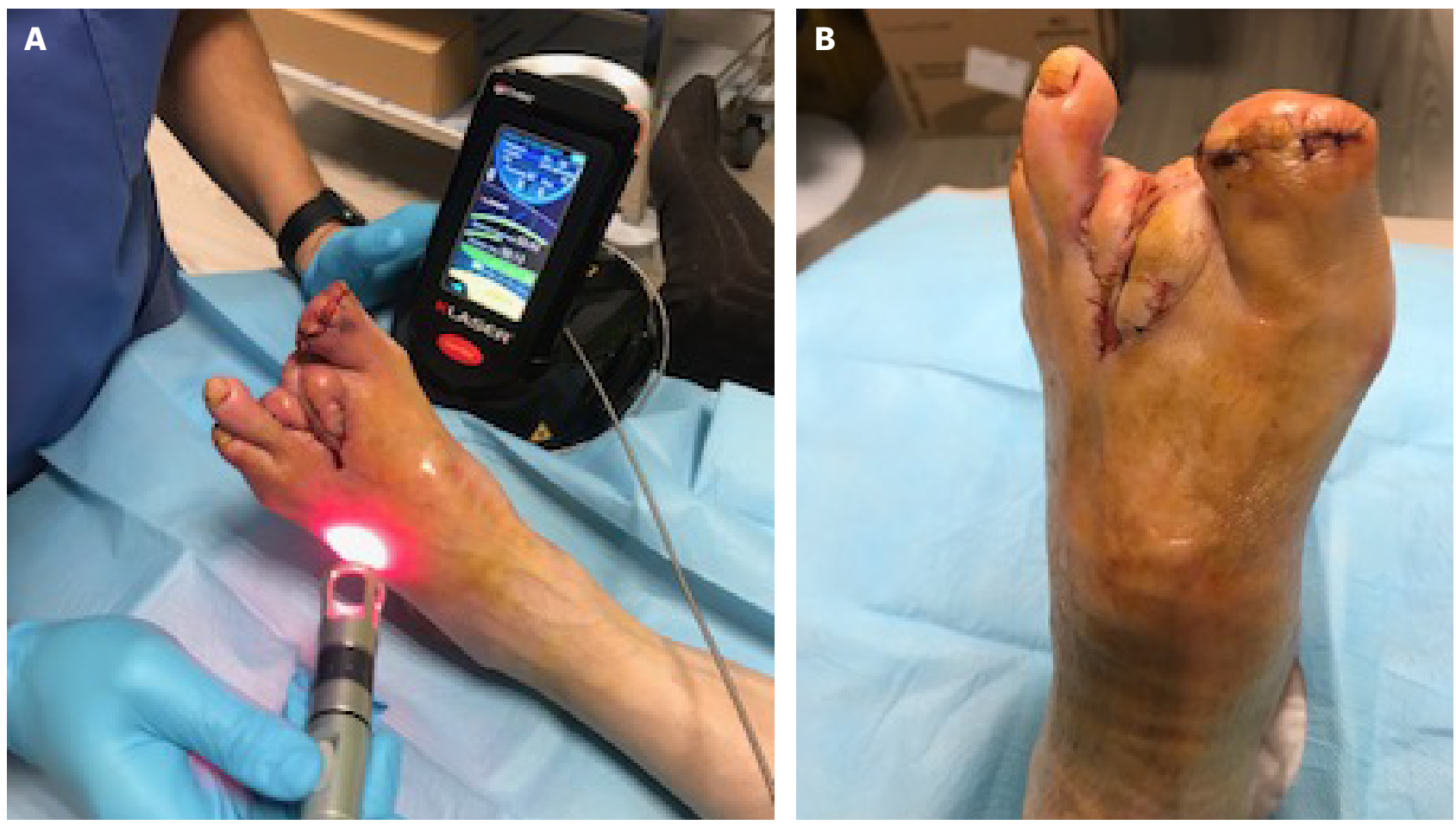

Figura 5. A. Sesión con láser cube 4. B. Resultado postoperatorio final.

que consistió en eliminar la matriz ungueal de forma completa junto a la mitad distal de la falange distal del dedo afectado, realizando un colgajo plantar y suturándolo en un solo plano a dorsal. Esta técnica ha demostrado, entre otras indicaciones, ser un tratamiento eficaz cuando se usa con prudencia para la gangrena distal del hallux y la osteomielitis asociada con la ulceración neuropática ${ }^{26}$.

Al paciente se le indicó que debía seguir con la misma pauta antibiótica, y se pautó desketoprofeno 25 mg (Enantyum ${ }^{\circledR}$, Menarini, España) cada 8 horas y omeprazol 20/24 horas una hora antes del desayuno, todo durante 14 días. A las $24 \mathrm{~h}$ de la intervención retomó su doble antiagregación tomando el clopidrogel $75 \mathrm{mg} / 24 \mathrm{~h}$, así como la enoxaparina, como tenía pautado previamente.

De manera postoperatoria se realizaron curas cada 3-4 días que consistieron en sesiones de K-laser Cube 4, ya que ha demostrado ser beneficioso como complemento de la terapia convencional en el tratamiento de las úlceras del pie diabético ${ }^{27,28}$, y en este caso, además, se observó mejoría del edema y dolor entre 0 y 1 después de su aplicación según escala EVA. Posteriormente se realizó cura tópica con undecilenamidopropil betaína, 0,1 \% polihexanida, glicerol, agua purificada e hidroxietilcelulosa (Prontosan gel ${ }^{\circledR}, \mathrm{B} /$ Braun, España) cubriéndolo con gasas con una capa de contacto antimicroniana (Sorbact ${ }^{\circledR}$, BSN Medical, España), hasta la cicatrización completa de las heridas quirúrgicas el 21 de noviembre de 2018, 5 semanas después de la intervención y tras 12 curas (Figura 5). El paciente siguió acudiendo cada mes y medio aproximadamente a consulta rutinaria hasta diciembre de 2019 sin incidencias reseñables.

\section{DISCUSIÓN}

El aumento de la esperanza de vida ha conllevado con ello un aumento de la prevalencia de las enfermedades crónicas como la isquemia crónica de extremidades inferiores, situada en nuestro medio en el 9,9 \% ${ }^{29,30}$. Se trata de una enfermedad muy invalidante generando restricción de la deambulación, dolor en reposo que mejora con la posición declive de las extremidades y/o lesiones tróficas en miembros inferiores que frecuentemente desencadenan una amputación mayor o menor del miembro ${ }^{31-33}$. Es una de las manifestaciones de la enfermedad ateroembólica, por lo que frecuentemente se acompaña de otras manifestaciones vasculares como la cardiopatía isquémica o la enfermedad cerebrovascular ${ }^{34,35}$ y presenta una incidencia más elevada en pacientes diabéticos o con insuficiencia renal crónica ${ }^{36,37}$.

Estas lesiones constituyen una consecuencia de una patología más extensa, por lo que además de valorar las lesiones, es importante hacer un estudio completo del estado vascular de la extremidad, puesto que sin flujo directo es improbable la curación de las lesiones ${ }^{38}$. Además de la necesidad o no de revascularización, es importante el tipo de cuidados que se realice, ya que este modifica la evolución de las lesiones y la frecuencia de amputación $n^{4,39,40}$. 
Larsson y cols., en 1995, ya habían demostrado la importancia del abordaje multidisciplinar de esta enfermedad ${ }^{16}$. Este caso es un ejemplo de este tipo de manejo. La colaboración de Enfermería, Podología, Cirugía vascular y Cirugía Plástica en este tipo de pacientes, junto con técnicas innovadoras, permite la resolución de lesiones de evolución tórpida que pueden requerir sucesivas amputaciones. Hay un aumento en la tasa de supervivencia de extremidades en pacientes que acuden a centros multidisciplinarios ${ }^{16,41,42}$. Esto ha sido resultado de avances en el cuidado del pie neuropático y del pie neuroisquémico, incluidos los avances en el manejo de infección ${ }^{43}$. No hay que olvidar la importancia que tiene en estos pacientes un seguimiento cercano del paciente, ya que los pacientes diabéticos que han desarrollado complicaciones crónicas de la diabetes tienen una mayor predisposición a las complicaciones postquirúrgicas ${ }^{44}$ y más aún en pacientes isquémicos ${ }^{40}$. Si cada miembro del equipo multidisciplinar desarrolla su labor de forma rápida, eficaz y hay una comunicación fluida entre los miembros de este equipo, estaremos más cerca de conseguir los objetivos propuestos de ser conservadores dentro del tratamiento del pie diabético, intentando mantener la mayor superficie posible del pie, ya que reduce el riesgo de recidivas y de la aparición de nuevas lesiones que predispongan al paciente a una nueva ulceración que aumente sus posibilidades de padecer otra osteomielitis y futura amputación que afecte a su esperanza y calidad de vida ${ }^{11}$.

Al paciente se le descartó para nuevas revascularizaciones desde el servicio de cirugía vascular por, lo que en este punto había que resolver un problema que consistía en una úlcera contaminada y compuesta por un muñón de amputación abierto que exponía la cabeza del tercer metatarsiano y dos articulaciones del segundo dedo. Ello requiere de una intervención quirúrgica lo menos agresiva posible, independientemente de que no se revascularice más, ya que en el pie afectado era necesaria una cobertura adecuada del esqueleto para evitar más infecciones.

Puesto que el segundo dedo es difícilmente salvable dado el grado de afectación que tiene (artritis séptica en las dos articulaciones interfalángicas, su desvascularización y exposición tendinosa), resulta razonable usarlo como "fillet toe" para cobertura del todo el defecto antes de que acabe necrosado completamente como consecuencia de la infección. Evidentemente, los riesgos de necrosis isquémica del colgajo son elevados, al menos de necrosis parciales, pero nos puede proporcionar una cobertura adecuada de un defecto mediano sin mucha morbilidad para el paciente, y con un colgajo que probablemente no dispondremos en el futuro si no intervenimos en la evolución de la úlcera, lo que nos abocaría invariablemente a una amputación mayor en este caso, donde las soluciones reconstructivas locorregionales son muy limitadas.

En conclusión, presentamos un caso clínico de úlcera neuroisquémica en un paciente con un perfil complejo, que fue tratado mediante un equipo multidisciplinar y que se resolvió de forma satisfactoria afortunadamente. Para llegar a este tipo de actuaciones es fundamental el trabajo multidisciplinar para evaluar las mejores opciones para los pacientes y que todos los miembros implicados estén al tanto de las actuaciones que se van a realizar y por qué, ya que en el supuesto que haya una complicación mayor y tenga que intervenir otro miembro del equipo como podría ser cirugía vascular, al conocer el caso de primera mano, los procedimientos de una amputación mayor estarían más indicados y el paciente lo aceptaría con una predisposición mayor ${ }^{7,15,45}$.

\section{CONFLICTO DE INTERESES}

Los autores declaran que el trabajo está libre de cualquier asociación personal o comercial que pueda suponer un conflicto de intereses en conexión con el artículo remitido, así como el haber respetado los principios éticos de investigación.

\section{FUENTES DE FINANCIACIÓN}

Ninguna.

\section{BIBLIOGRAFÍA}

1. Viadé J. Pie diabético. Guía práctica para la prevencion, evaluacion y

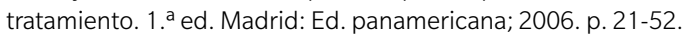

2. Jakosz N. Book review - IWGDF Guidelines on the Prevention and Management of Diabetic Foot Disease. Wound Pract Res. 2019. DOI: 10.33235/wpr.27.3.144

3. Tracey ML, Gilmartin M, O'Neill K, Fitzgerald AP, McHugh SM, Buckley $\mathrm{CM}$, et al. Epidemiology of diabetes and complications among adults in the Republic of Ireland 1998-2015: A systematic review and meta-analysis. BMC Public Health. 2016; 16(1): 132. DOI: 10.1186/s12889-0162818-2.

4. Boulton AJM, Vileikyte L, Ragnarson-Tennvall G, Apelqvist J. The global burden of diabetic foot disease. Lancet. 2005;366(9498):1719-24. DOI: 10.1016/S0140-6736(05)67698-2.

5. Montiel-Jarquín AJ, García Villaseñor A, Castillo Rodríguez C, Romero-Figueroa MS, Etchegaray-Morales I, García-Cano E, et al. Costes directos de atención médica del pie diabético en el segundo nivel de atención médica. Rev Chil Cirugía. 2017;69(2):118-23. DOI: 10.1016/j. rchic.2016.09.009.

6. Armstrong DG, Boulton AJM, Bus SA. Diabetic foot ulcers and their recurrence. N Engl J Med. 2017;376(24):2367-75. DOI: 10.1056/ NEJMra1615439.

7. Edmonds M. Infection in the neuroischemic foot. Int J Low Extrem Wounds. 2005;4(3):145-53. DOI: 10.1177/1534734605280597.

8. Pedras S, Carvalho R, Pereira MDG. Sociodemographic and clinical characteristics of patients with diabetic foot ulcer. Rev Assoc Med Bras (1992). 2016;62(2):171-8. DOI: 10.1590/1806-9282.62.02.171.

9. Rutherford RB, Baker JD, Ernst C, Johnston KW, Porter JM, Ahn S, et al. Recommended standards for reports dealing with lower extremity ischemia: Revised version. J Vasc Surg. 1997;26(3):517-38. DOI: 10.1016/ S0741-5214(97)70045-4.

10. García Herrera AL. El pie diabético en cifras. Apuntes de una epidemia. Rev Médica Electrónica. 2016;38(4).

11. Pecoraro RE, Reiber GE, Burgess EM. Pathways to Diabetic Limb Amputation Basis for Prevention An identifiable and potentially preventable pivotal event, in most cases an episode involving minor. Diabetes Care. 1990;13(5):513-21. DOI: 10.2337/diacare.13.5.513. 
12. Prompers L, Huijberts M, Apelqvist J, Jude E, Piaggesi A, Bakker K, et al. High prevalence of ischaemia, infection and serious comorbidity in patients with diabetic foot disease in Europe. Baseline results from the Eurodiale study. Diabetologia. 2007;50(1):18-25. DOI: 10.1007/ s00125-006-0491-1.

13. Morbach S, Furchert H, Gröblinghoff U, Hoffmeier H, Kersten K, Klauke GT, et al. Long-term prognosis of diabetic foot patients and their limbs: Amputation and death over the course of a decade. Diabetes Care. 2012;35(10):2021-7. DOI: 10.2337/dc12-0200.

14. Dargis V, Pantelejeva O, Jonushaite A, Vileikyte L, Boulton AJM. Benefits of a multidisciplinary approach in the management of recurrent diabetic foot ulceration in Lithuania: A prospective study. Diabetes Care. 1999;22(9):1428-31. DOI: 10.2337/diacare.22.9.1428.

15. Hingorani A, Lamuraglia GM, Henke $P$, Meissner MH, Loretz L, Zinszer $\mathrm{KM}$, et al. The management of diabetic foot: A clinical practice guideline by the Society for Vascular Surgery in collaboration with the American Podiatric Medical Association and the Society for Vascular Medicine. J Vasc Surg. 2016;63(2 Suppl):3S-21S. DOI: 10.1016/j.jvs.2015.10.003.

16. Larsson J, Apelqvist J, Agardh CD, Stenström A. Decreasing Incidence of Major Amputation in Diabetic Patients: a Consequence of a Multidisciplinary Foot Care Team Approach? Diabet Med. 1995; 12(9):770-6. DOI: 10.1111/j.1464-5491.1995.tb02078.x.

17. IMI. CARE case report guidelines. CARE Case Report Guidelines.

18. Sohrabi S, Russell D. Diabetic foot and foot debridement technique. Surg (United Kingdom). 2017;35(9):500-4. DOI: 10.1016/j. mpsur.2017.06.009.

19. Melzack R, Katz J, Jeans ME. The role of compensation in chronic pain: Analysis using a new method of scoring the McGill Pain Questionnaire. Pain. 1985;23(2):101-12. DOI: 10.1016/0304-3959(85)90052-1.

20. Buendía PérezJ, Vila Sobral A, Gómez Ruiz R, Qiu Shao SS, Marré Medina D, Romeo M, et al. Tratamiento de heridas complejas con terapia de presión negativa. Experiencia en los últimos 6 años en la Clínica Universitaria de Navarra, Pamplona (España). Cir Plast Iberolatinoam. 2011;37(Supl.1). DOI: 10.4321/S0376-78922011000500010.

21. Berlanga-Acosta J, Gavilondo-Cowley J, López-Saura P, et al. Epidermal growth factor in clinical practice - A review of its biological actions, clinical indications and safety implications. Int Wound J. 2009;6(5):331-46. DOI: 10.1111/j.1742-481X.2009.00622.x.

22. Lavery LA, Armstrong DG, Peters EJG, Lipsky BA. Probe-to-bone test for diagnosing diabetic foot osteomyelitis: Reliable or relic? Diabetes Care. 2007;30(2):270-4. DOI: 10.2337/dc06-1572.

23. Attinger CE, Venturi M, Kim K, Ribiero C. Maximizing length and optimizing biomechanics in foot amputations by avoiding cookbook recipes for amputation. Semin Vasc Surg. 2003;16(1):44-66. DOI: 10.1053/ svas.2003.50006.

24. Vivas D, Roldán I, Ferrandis R, Marín F, Roldán V, Tello-Montoliu A, et al. Perioperative and Periprocedural Management of Antithrombotic Therapy: Consensus Document of SEC, SEDAR, SEACV, SECTCV, AEC, SECPRE, SEPD, SEGO, SEHH, SETH, SEMERGEN, SEMFYC, SEMG, SEMICYUC, SEMI, SEMES, SEPAR, SENEC, SEO, SEPA, SERVEI, SECOT and AEU. Rev Esp Cardiol (Engl Ed). 2018;71(7):553-64. DOI: 10.1016/j.rec.2018.01.029.

25. Chung SR, Wong KL, Cheah AEJ. The lateral lesser toe fillet flap for diabetic foot soft tissue closure: Surgical technique and case report. Diabet Foot Ankle. 2014;5:25732. DOI: 10.3402/dfa.v5.25732.

26. Boffeli TJ, Goss MS. Distal Syme Hallux Amputation for Tip of Toe Wounds and Gangrene Complicated by Osteomyelitis of the Distal Phalanx: Surgical Technique and Outcome in Consecutive Cases. J Foot Ankle Surg. 2018;57(3):456-61. DOI: 10.1053/j.jfas.2017.10.027.

27. Mathur RK, Sahu K, Saraf S, Patheja P, Khan F, Gupta PK. Low-level laser therapy as an adjunct to conventional therapy in the treatment of diabetic foot ulcers. Lasers Med Sci. 2017;32(2):275-82. DOI: 10.1007/ s10103-016-2109-2.
28. Kajagar BM, Godhi AS, Pandit A, Khatri S. Efficacy of Low Level Laser Therapy on Wound Healing in Patients with Chronic Diabetic Foot Ulcers-A Randomised Control Trial. Indian J Surg. 2012;74(5):359-63. DOI: 10.1007/s12262-011-0393-4.

29. Vallina-Vázquez MJ, Vaquero-Lorenzo F, Álvarez-Salgado A, et al. A study of the prevalence of chronic ischaemia in the lower limbs and abdominal aortic aneurysms in persons over the age of 65 . Angiologia. 2007;59(3):225-35. DOI: 10.1016/S0003-3170(07)75049-6.

30. Fowkes FGR, Rudan D, Rudan I, Aboyans V, Denenberg JO, McDermott $M M$, et al. Comparison of global estimates of prevalence and risk factors for peripheral artery disease in 2000 and 2010: A systematic review and analysis. Lancet. 2013;382(9901):1329-40. DOI: 10.1016/S01406736(13)61249-0.

31. Miñano Pérez A, Jiménez-Gil R, Ortiz-De Saracho M, Villanueva V, Bernabeu-Pascual F, González-Gutiérrez MA, et al. Realidad social de los pacientes con isquemia crítica de miembros inferiores. Angiologia. 2008;60(4):241-5. DOI: 10.1016/s0003-3170(08)04002-9.

32. Hernando F, Ducajú G. Isquemia de los miembros inferiores. En: Libro de la salud cardiovascular del Hospital Clínico San Carlos y la Fundación BBVA. San Sebastián: Editorial Nerea; 2009. p. 519-26. Disponible en: http://www.fbbva.es/TLFU/microsites/salud_cardio/mult/fbbva_ libroCorazon_cap59.pdf.

33. Aristizábal Londoño P, Duque Yepez M, Ortega Gaviria M, Berbesi Fernández D. Caracterización de pacientes con Isquemia Crítica Crónica de miembros inferiores. Rev CES Salud Pública. 2012;3(1):18-27. DOI: $10.21615 / 2073$.

34. Fernández Travieso JC. Enfermedad arterial periférica en adultos mayores. Rev CENIC Ciencias Biológicas. 2013;44(3):1-13.

35. Serrano Hernando FJ, Conejero AM. Peripheral artery disease: Pathophysiology, diagnosis and treatment. Rev Esp Cardiol. 2007.;60(9):96982. DOI: 10.1157/13109651.

36. Marrón B, Coronel F, López-Bran E, Barrientos A. Calciphylaxis: an uncertain pathogenesis and controversial treatment. Nefrologia. 2001;21(6):596-600.

37. Sender Palacios MJ, Vernet Vernet M, Larrosa Sáez P, Tor Figueras E, Foz Sala M. Socio-demographic and clinical characteristics of a patient population with diabetes mellitus. Aten Primaria. 2002.;29(8):474-80 DOI: 10.1016/S0212-6567(02)70616-1.

38. Tendera M, Aboyans V, Bartelink ML, Baumgartner I, Clément D, Collet JP, et al. ESC Guidelines on the diagnosis and treatment of peripheral artery diseases. Eur Heart J. 2011.;32(22):2851-906. DOI: 10.1093/ eurheartj/ehr211.

39. American Diabetes Association. Preventive Foot Care in Diabetes. Diabetes Care. 2004;27(1):63-4. DOI: 10.2337/diacare.27.2007.s63.

40. Jeffcoate WJ, Van Houtum WH. Amputation as a marker of the quality of foot care in diabetes. Diabetologia. 2004.;47(12):2051-8. DOI: 10.1007/s00125-004-1584-3.

41. Faglia E, Favales F, Aldeghi A, et al. Change in major amputation rate in a center dedicated to diabetic foot care during the 1980s: Prognostic determinants for major amputation. J Diabetes Complications. 1998;12(2):96-102. DOI: 10.1016/S1056-8727(97)98004-1.

42. Holstein P, Ellitsgaard N, Bornefeldt Olsen B, Ellitsgaard V. Decreasing incidence of major amputations in people with diabetes. In: Vasa - J Vascular Diseases. 2001. DOI: 10.1024/0301-1526.30.s58.28.

43. Edmonds ME, Foster AVM. Reduction of major amputations in the diabetic ischaemic foot: A strategy to "take control" with conservative care as well as revascularisation. In: Vasa - J Vascular Diseases. 2001. DOI: 10.1024/0301-1526.30.s58.6

44. Varma AK. Reconstructive foot and ankle surgeries in diabetic patients. Indian J Plast Surg. 2011;44(3):390-5. DOI: 10.4103/0970-0358.90806.

45. Korzon-Burakowska A, Dziemidok P. Diabetic foot - the need for comprehensive multidisciplinary approach. Ann Agric Environ Med. 2011;18(2):314-7. 